\title{
Estudio preliminar de la adquisición de conocimientos de la concienciación ecológica en la educación secundaria obligatoria: la educación ambiental entre los adolescentes del nordeste de España
}

\author{
Meritxell Notari Llorens, Universidad Miguel Hernández, España \\ Francisco Pardo Fabregat, Universidad CEU Cardenal Herrera, España \\ Manuel Jordán Vidal, Universidad Miguel Hernández, España
}

\begin{abstract}
Resumen: Actualmente se han detectado carencias en la educación ambiental aun estando viviendo momentos de cambio global. En el presente estudio de investigación, se examina la adquisición de los conocimientos relacionados con la concienciación ecológica en los alumnos de educación secundaria en el nordeste de España. Se realizó un cuestionario para cada curso con diferentes tipos de preguntas: especificas de curso y conocimientos previos. Tras un análisis estadístico, con el lenguaje y entorno de programación para análisis estadístico $R$, se realizó un contraste de medias unilateral donde se contrastó la media de respuestas respondidas correctamente de los alumnos de los diferentes cursos de educación secundaria, a final y a inicio de curso, de dicho cuestionario. Tras el análisis de los resultados se observa que la media de respuestas respondidas correctamente a final de curso es mayor que la media al inicio de curso en la mayoría de casos estudiados a excepción de dos grupos. Las conclusiones de este estudio indican que la educación que reciben nuestros alumnos concluye en un aprendizaje significativo tal y como se indica aunque no debemos descuidar la formación no formal que reciben los alumnos para así tener ciudadanos concienciados con el medio ambiente.
\end{abstract}

Palabras clave: ciencias naturales, educación formal, concienciación ecológica

\begin{abstract}
Nowadays lacks in environmental education were detected even living in times of global change. In this research, the acquisition of knowledge related to environmental consciousness in secondary school students in northeast Spain is examined. A questionnaire for each secondary course was made with different kind of questions: specific of the course and of the previous knowledge. After a statistical analysis, with R programming language, a unilateral hypothesis contrast where the mean responses of the students answered correctly at the beginning and the end of the different courses of secondary education, were contrasted. After analysis, the results shows that the mean responses answered correctly at the end of the course is greater than the mean at the beginning of course in most cases studied, except two groups. The findings of this study indicate that the education of our students conclude with a meaningful learning as indicated but we should not neglect the non-formal education received by students in order to have people environmentally conscious.
\end{abstract}

Keywords: Natural Science, Formal Education, Ecological Consciousness

\section{Introducción}

$\mathrm{L}$ a conciencia ambiental (o ecológica) se asocia al llamado nuevo paradigma ambiental (o ecológico). Este paradigma asocia ambientalismo a una visión del mundo eco-céntrica que hace hincapié en la necesidad humana de establecer un equilibrio con la naturaleza, establecer la existencia de límites de crecimiento a la sociedad y cuestionar el derecho de la humanidad de gobernar el resto de la naturaleza (Jiménez y Lafuente, 2010).

La preocupación por el medio ambiente no es una cuestión reciente sino que ya en las últimas décadas hubo muchos eventos que se han preocupado de cómo la sobreexplotación de la actividad socioeconómica está más allá de la capacidad del ecosistema para generar recursos y

Revista Internacional de Aprendizaje en Ciencia, Matemáticas y Tecnología

Volumen 3, Número 2, 2016, <http://sobrelaeducacion.com>, ISSN 2386-8791

(C) Global Knowledge Academics. Meritxell Norati, Francisco Pardo y Manuel Jordán.

Todos los derechos reservados. Permisos: soporte@gkacademics.com 
absorber residuos. Ejemplos de esta preocupación son diferentes libros como "Silent spring" (Carson, 1962), "The limits to grow" (Meadows, Meadows, Randers y Behrens, 1972), " The Closing Circle. Nature, Man and Technology" (Commoner, 1971) o "The small is beautiful" (Schumacher, 1973), en ellos se reflexiona, analiza y criticar el desarrollo excesivo, el crecimiento económico y la poca preocupación por el medio ambiente y la conciencia ecológica.

Por otra parte, diferentes instituciones y organizaciones internacionales, como la ONU, a principios de los años sesenta comienzan a promover diferentes programas, conferencias y seminarios con el fin de tomar decisiones relacionadas con la educación ambiental y la conciencia ecológica, y hacer que estos conceptos y estrategias sean disponibles a toda la sociedad (Alonso, 2010).

En las instituciones españolas el desarrollo de conferencias y programas se inició en los años setenta, este inicio está marcado por dos libros "El bosc de Santiga" y "Ecología y Educación Ambiental" (Benayas, Gutiérrez, \& Hernández, 2003). La historia española de la educación ambiental se resume en el "Libro Blanco de la Educación Ambiental en España", publicado en 1999 (AA.VV., 1999). En el año 2001 se analizaron los hábitos y actitudes de la sociedad española, en este estudio se indica que la población española es consciente de la necesidad de proteger el medio ambiente y que, dicha protección, debe estar por encima del desarrollo económico y los hábitos de consumo, por esto la población española se puede definir como pro-ecologista (Sánchez, 2001) . Con este estudio se demuestra que los esfuerzos por parte de las autoridades no fueron en vano. Dentro de esta perspectiva de respeto y conscientes de nuestro entorno, debemos trabajar, enseñar y educar, dentro y fuera del aula, a las generaciones más jóvenes fomentando de esta manera la conciencia ecológica, tal y como indican Boyes y Stanisstreet (Boyes y Stanisstreet, 2012).

\section{Marco teórico: la conciencia ecológica en los centros de educación secundaria}

La educación ambiental en los centros de educación secundaria es un tema muy analizado tanto en España como en todo el mundo. En España estos estudios analizan la formación inicial del profesor de educación secundaria (Moreno, 2005), también se estudian las creencias y deficiencias de los maestros de la educación primaria en relación con la educación ambiental como un punto crítico para su implementación en el currículo (Nando, 1995) u otros estudios en los que se analiza la evolución de la práctica docente y su formación a largo plazo (Vázquez-Bernal, Mellado, Jiménez-Pérez y Leñero, 2012). Otros estudios se centran en los estudiantes y su conocimiento de la naturaleza como el estudio propuesto por Jaén y Barbudo (Jaén y Barbudo, 2010), donde se estudia la evolución de las percepciones sobre el medio ambiente en un año académico basándose en la escala ENV -escala para medir las percepciones ambientales, tanto en la actitud y el comportamiento, propuestas por Bogner y Wilhelm en 1996 (Bogner \& Wilhelm, 1996)- donde se detectaron diferencias de las actitudes de los estudiantes educación secundaria, como resultado de un trabajo monográfico (Pérez, Pérez y Quijano, 2009). Otros compilan diferentes trabajos con el fin de tener una visión amplia de temas Socio-Científicos (Tytler, 2012). Hay estudios en los que el autor analiza el modelo de enseñanza y sugiere propuestas de mejora para la educación ambiental (Reategui, 1999), también otros trabajos donde se compilan diferentes estrategias para mejorar la educación ambiental (Garcia y Nando, 2000) u otros estudios en los que se examina la motivación de los estudiantes - de 14 a 16 años de edad - en aprender ciencia y la forma en que el Advanced Placement Program (AP) y de cómo el aprendizaje colaborativo en ciencia pueden cambiar la motivación del estudiante por aprender la ciencia (Bryan, Glynn, \& Kittleson, 2011). También se analizaron los informes del Programme for International Student Assessment (PISA) recogen las actitudes y la responsabilidad de los adolescentes hacia el medio ambiente (OECD, 2012a, 2012b) y otros estudios analizan los datos de los informes PISA para asi cuantificar el grado de compromiso con la ciencia en los adolescentes de 15 años de edad (Hampden-Thompson y Bennett, 2013). 


\section{El presente estudio}

La educación ambiental y los conceptos de conciencia ecológica en la educación formal en España son definidos por el gobierno. La legislación vigente para el período en que se realizó este trabajo era la "Ley Orgánica de Educación 2 / 2006" (Boletín Oficial del Estado, 2006a), esta ley indica las capacidades que el estudiante de educación secundaria (edades entre doce a dieciséis) debe adquirir durante este período, estas capacidades se definen y se recogen en el "Real Decreto 1636/2006" (Boletín Oficial del Estado, 2006b) a nivel nacional y por el DOCV 112/2007 (Generalitat Valenciana, 2007) a nivel regional. Si se analiza la legislación, se puede observar que los conceptos relacionados con la conciencia ecológica se encuentran dispersos en todo el período de la educación secundaria. Esto es indicativo de la temática transversal de estos conceptos y el esfuerzo que implica la introducción de una temática transversal dentro de una estructura ya consolidada (Gavidia, 2000). A pesar de dicha transversalidad, es la asignatura de biología la que contribuye de manera principal en la adquisición de conceptos conciencia ecológica. Con el marco teórico definido, el presente trabajo tiene como objetivo analizar la adquisición de los conceptos relacionados con la conciencia ecológica por los alumnos de educación secundaria en el noreste de España, en la provincia de Castellón dividida en ocho comarcas (figura 1).

Figura 1: Localización geográfica y comarcas de la provincial de Castellón (nordeste de España).

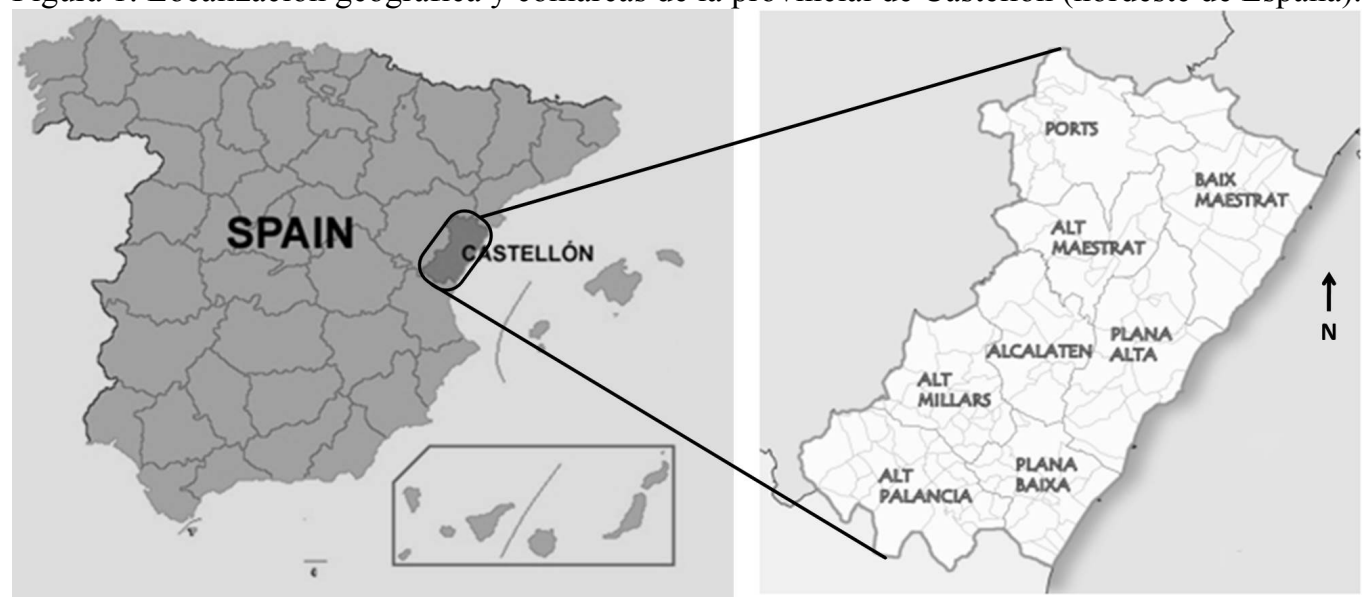

\section{Objetivo}

El objetivo de este estudio es evaluar y cuantificar la adquisición de los conocimientos, relacionados con la concienciación ecológica propuestos por la legislación vigente, por parte de los alumnos de educación secundaria en la provincia de Castellón, España. Para conseguir este objetivo se compararon las diferencias entre los conceptos previos y los adquiridos a lo largo del mismo curso académico.

\section{Metodología}

La metodología es uno de los puntos clave en la elaboración de un trabajo de investigación. La metodología utilizada en el presente trabajo es relativa a las ciencias de la educación bajo un punto de vista de las ciencias sociales (Figura 2). 
Figura 2: Diagrama de flujo de la metodología seguida en esta investigación.

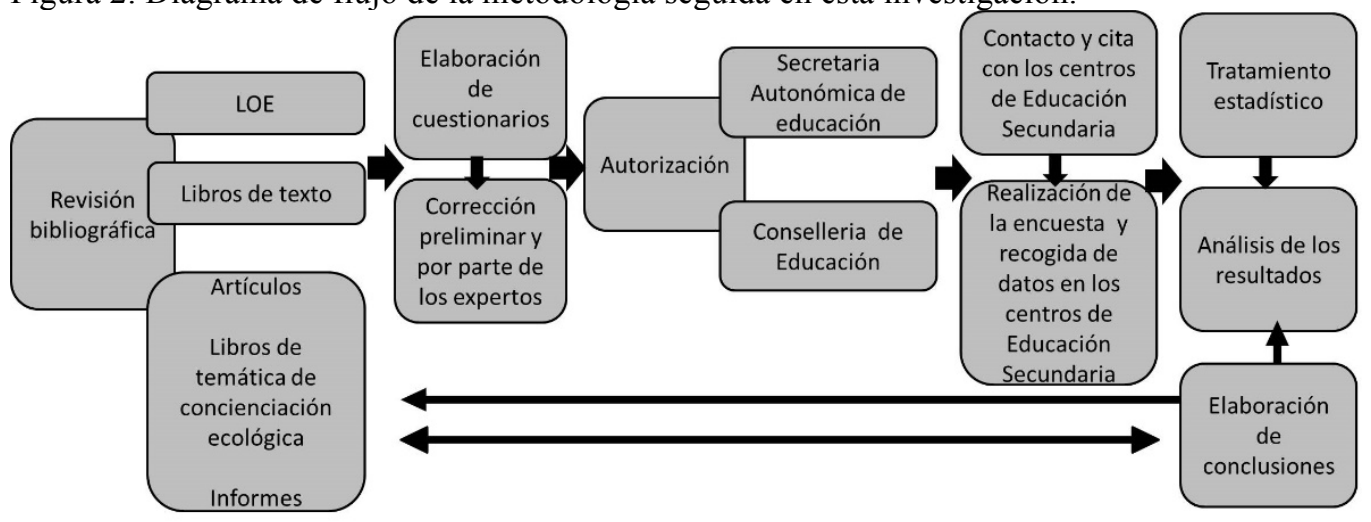

Se realizó una búsqueda bibliográfica para así establecer el marco teórico, posteriormente se procede a la elaboración del cuestionario con el cual se pretende conocer el conocimiento de en conciencia ecológica de los estudiantes de educación secundaria. Para realizar este trabajo, es necesaria la autorización por la Conselleria de Educación de la Generalitat Valenciana, por la característica de la población estudiada. Tras contactar con los centros de educación secundaria, se acuerda una fecha para realizar el cuestionario a los estudiantes, recoger los datos para así procesar los resultados, analizarlos y obtener las conclusiones.

\section{Elaboración del cuestionario}

La principal herramienta para recoger los datos de la población estudiada, en este trabajo los estudiantes de educación secundaria de la provincia de Castellón, es el cuestionario. El proceso de elaboración del cuestionario debe ser fiable con el fin de "obtener de manera sistemática información de la población investigada sobre las variables objeto de la investigación" (Sierra, 1998).

En la elaboración del cuestionario de los alumnos se realizó una sistematización de los formularios, es decir, cada uno de los alumnos debían responder al mismo cuestionario basado en la revisión bibliografía realizada. El tipo de cuestionario era de tipo test con tres respuestas posibles donde solo una opción era correcta. Se eligió en este caso ser tipo test para que su corrección fuese lo más objetiva posible y que las creencias y/o experiencias personales no influyesen en el momento del análisis de los resultados.

Cada curso respondía a un número diferente de preguntas, adaptándola así al curso de educación secundaria correspondiente. Con este tipo de cuestionario, se tiene diferentes preguntas con las cuales obtenemos distinta información. Se diferencia así las preguntas de conocimientos previos, las preguntas específicas de curso que conforman los conocimientos adquiridos y la totalidad de los conocimientos que poseen los alumnos. La siguiente tabla (tabla 1) muestra de una forma visual el número de preguntas y el tipo de las mismas planteadas en el cuestionario dirigido a los alumnos.

Tabla 1: Tipo y número de preguntas del cuestionario para cada curso de educación secundaria

\begin{tabular}{|c|c|c|c|}
\hline $\begin{array}{ll}\text { Curso } & \text { Tipo de preguntas } \\
\end{array}$ & Previas & Especificas & Total \\
\hline $1^{\circ}$ & ------ & 19 & 19 \\
\hline $2^{\circ}$ & 19 & 8 & 27 \\
\hline $3^{\circ}$ & 27 & 9 & 36 \\
\hline $4^{\circ}$ & 36 & 5 & 41 \\
\hline
\end{tabular}




\section{Población estudiada}

La principal característica del presente trabajo es que, la población estudiada, son estudiantes de entre 12 a 16 años por lo que son menores de edad y están protegidos por ley. Por este motivo, cualquier cuestionario para trabajos de investigación que realice el alumno de cualquier centro decente debe estar autorizado por la Conselleria de Educación en este caso particular, por la Secretaria Autonómica de Educación. Si bien que en el área de didáctica de las ciencias experimentales, en la etapa educación secundaria, existen diversos trabajos que evalúan la evolución de las percepciones medioambientales de los alumnos de educación secundaria como el trabajo de Jaén y Barbudo (Jaén \& Barbudo, 2010) o el de Castells y Morey (Castells y Morey, 2004), en los cuales no hay constancia de que se pida autorización a las autoridades pertinentes.

\section{Sistematización de los datos}

Tras la recogida de datos, es necesaria una correcta sistematización para conservar la objetividad, imparcialidad y coherencia de los datos. La principal aproximación es la correcta elaboración y mejora del cuestionario. Además los estudiantes respondían al cuestionario en una plantilla, común a todos los estudiantes, alcanzando así una estandarización y sistematización optima de los datos recogidos.

\section{Análisis estadístico}

Tras la sistematización de los datos, se procede al análisis de los resultados. Este análisis es estadístico bajo un punto de vista de las ciencias sociales (Peña, 1997).

Los cálculos estadísticos, en la actualidad, están facilitados gracias a la accesibilidad de los ordenadores y el desarrollo de programas informáticos y estadísticos fáciles de usar y con una interfaz intuitiva para el usuario. Este desarrollo permite una sencilla aplicación de la estadística en un amplio rango de los diferentes campos de la investigación. Aunque estos campos sean diferentes, los métodos estadísticos que se utilizan son similares. La estandarización de los métodos estadísticos que se utilizan ha facilitado la aparición de diferentes paquetes estadísticos con diferentes aspectos como la capacidad, su facilidad de uso o el entorno de trabajo. Estos paquetes estadísticos son accesibles a los investigadores que necesitan realizar análisis estadísticos sencillos para su trabajo.

Entre estos paquetes se encuentran el Statistical Package for the Social Sciences (SPSS) (IBM Corp. Released, 2012) y el lenguaje y entorno de programación R (R Core Team, 2014). Cada paquete estadístico posee diferentes características, cualidades y capacidades analíticas motivo por el cual se han utilizados dos programas diferentes para realizar el análisis estadístico de los datos recogidos en los diferentes cuestionarios

El paquete estadístico SPSS es un sistema amplio, flexible y fácil de usar con un interface muy intuitiva de análisis estadístico y de gestión de datos. Asimismo posee una amplia difusión a diferentes niveles, tanto educativo como profesional, pero por el contrario posee un número limitado de paquetes estadísticos, que aunque con versiones posteriores se actualizan, limitan su uso cuando es necesario realizar un análisis estadístico más exhaustivo. Por el contrario, posee unos módulos, como variables estadísticas descriptivas elementales, análisis de frecuencias o medias que son muy valiosos para realizar la estadística descriptiva.

Por otra parte, el lenguaje y entorno de programación R, proporciona un amplio abanico de herramientas estadísticas y gráficas y al tratarse de un lenguaje de programación el usuario puede definir sus propias funciones. $\mathrm{R}$ es más que un software para el análisis de datos, es un entorno de trabajo que va incrementando continuamente sus capacidades con la incorporación de paquetes y funciones que se integran perfectamente en el sistema R (Elosua, 2009). Es por esto que posee unas cualidades que le hacen ser un lenguaje y entorno de programación más robusto para 
el contraste de hipótesis comparado con SPSS, es decir, R ofrece mayores garantías en los contrastes de hipótesis.

Por estas cualidades, antes mencionadas, se utilizaron ambos programas estadísticos para el análisis e interpretación de los resultados recopilados. Por una parte se utilizó el programa SPSS para realizar el análisis estadístico descriptivo y por otra parte se utilizó el lenguaje y entorno de programación R para realizar los contrastes de hipótesis.

\section{Resultados y discusión}

\section{Estadística descriptiva}

La población estudiada, los alumnos de educación secundaria, está localizada en el nordeste de España, en la provincia de Castellón. Esta provincia está dividida en ocho comarcas. Relativamente a las comarcas, un total de 35 y 19 centros de educación secundaria fueron encuestados, al inicio y al final del curso académico (tabla 2). Los alumnos se pueden dividir según diferentes parámetros como el tipo de centro de educación secundaria (público, privado o concertado) (tabla 3), según el curso de educación secundaria que cursa el alumno (de primero a cuarto) (tabla 4) o según el género (tabla 5). Un total de 4740 y 2520 estudiantes fueron encuestados al inicio y al fin del curso académico. Como se puede observar, la muestra de la población estudiada, reúne todas las características de la población, esto demuestra que la muestra es representativa. Por este motivo, el presente trabajo representa el inicio para un análisis estadístico de otras poblaciones con características similares a la aquí presentada.

Tabla 2: Centros de educación secundaria encuestados al inicio y al final de curso académico según las comarcas

\begin{tabular}{|c|c|c|}
\hline & \multicolumn{2}{|c|}{ Curso académico } \\
\hline Comarcas & Inicio & Fin \\
\hline Els Ports & 1 & 1 \\
\hline Alt Maestrat & 3 & 2 \\
\hline Baix Maestrat & 3 & 1 \\
\hline Alcalatén & 4 & 2 \\
\hline Alt Millars & 1 & 1 \\
\hline Alt Palancia & 3 & 2 \\
\hline Plana Alta & 8 & 5 \\
\hline Plana Baixa & 12 & 5 \\
\hline Total & 35 & 19 \\
\hline
\end{tabular}

Tabla 3: Estudiantes encuestados al inicio y final del curso académico según el tipo de centro de educación secundaria. Frec significa frecuencia y Pct significa porcentaje.

\begin{tabular}{|c|c|c|c|c|}
\hline \multirow{2}{*}{ Tipo de centro de educación secundaria } & \multicolumn{4}{|c|}{ Estudiantes encuestados } \\
\cline { 2 - 5 } & \multicolumn{2}{|c|}{ Inicio } & \multicolumn{2}{|c|}{ Fin } \\
\hline & Frec & Pct & Frec & Pct \\
\hline Públicos & 3113 & $65,7 \%$ & 1268 & $50,3 \%$ \\
\hline Concertados & 1527 & $32,2 \%$ & 1136 & $45,1 \%$ \\
\hline Privados & 100 & $2,1 \%$ & 116 & $4,6 \%$ \\
\hline TOTAL & 4740 & $100 \%$ & 2520 & $100 \%$ \\
\hline
\end{tabular}


Tabla 4: Estudiantes encuestados al inicio y final del curso académico según el curso de educación secundaria. Frec significa frecuencia y Pct significa porcentaje

\begin{tabular}{|c|c|c|c|c|}
\hline \multirow{2}{*}{ Curso de educación secundaria } & \multicolumn{3}{|c|}{ Estudiantes encuestados } \\
\cline { 2 - 5 } & \multicolumn{2}{|c|}{ Inicio } & \multicolumn{2}{|c|}{ Fin } \\
\hline & Frec & Pct & Frec & Pct \\
\hline $1^{\mathrm{o}}$ & 1429 & $30^{\prime} 1 \%$ & 792 & $31^{\prime} 1 \%$ \\
\hline $2^{\mathrm{o}}$ & 1315 & $27^{\prime} 7 \%$ & 664 & $26^{\prime} 7^{\prime} \%$ \\
\hline $3^{\mathrm{o}}$ & 1198 & $25^{\prime} 3 \%$ & 621 & $24^{\prime} 6 \%$ \\
\hline $4^{\mathrm{o}}$ & 798 & $16^{\prime} 8 \%$ & 443 & $17^{\prime} 6 \%$ \\
\hline TOTAL & 4740 & $100 \%$ & 2520 & $100 \%$ \\
\hline
\end{tabular}

Tabla 5: Estudiantes encuestados al inicio y final del curso académico según el género (masculino o femenino). Frec significa frecuencia y $P c t$ significa porcentaje

\begin{tabular}{|c|c|c|c|c|}
\hline \multirow{2}{*}{ Género } & \multicolumn{3}{|c|}{ Estudiantes encuestados } \\
\cline { 2 - 5 } & \multicolumn{2}{|c|}{ Inicio } & \multicolumn{2}{c|}{ Fin } \\
\hline & Frec & Pct & Frec & Pct \\
\hline Masculino & 2276 & $48 \%$ & 1195 & $47^{\prime} 4 \%$ \\
\hline Femenino & 2464 & $52 \%$ & 1325 & $52^{\prime} 6 \%$ \\
\hline TOTAL & 4740 & $100 \%$ & 2520 & $100 \%$ \\
\hline
\end{tabular}

\section{Contraste de Hipótesis}

Para alcanzar el objetivo propuesto en este estudio, se realizó un contraste de hipótesis que fue testada con el lenguaje de programación R. Previamente al contraste, la muestra fue analizada para verificar que la misma cumple con los requisitos de aplicabilidad requeridos para realizar el contraste de hipótesis. Las medias se compararon utilizando test-t independientes y se consideraron significativas cuando el p-valor era $<0.05$. (Bryan et al., 2011; Stevens, 2012).

El contraste unilateral que se diseñó trata de responder a la siguiente pregunta: ¿la media de preguntas respondidas correctamente al final del curso académico es mayor que la media de preguntas respondidas correctamente al inicio del curso académico? Con este contraste de hipótesis, se compara toda la información que es posible extraer del formulario, es decir, los conceptos previos, específicos y totales.

Tal y como indican los resultados (tabla 6), tenemos evidencia estadística suficiente para rechazar la hipótesis nula en la mayoría de los casos. Es decir, la media de preguntas respondidas correctamente a final del curso académico es mayor que la media de preguntas respondidas correctamente a inicio del curso académico. Por esto, los estudiantes de educación secundaria de la provincia de Castellón (nordeste de España) realizan un aprendizaje significativo de los conceptos relacionados con la concienciación ecológica propuestos por legislación. Este resultado es coherente con el informe PISA (OECD, 2012b) donde se indica que la principal fuente de información relacionada con el medio ambiente es el centro de estudios. En este estudio se cuantifica y verifica mediante métodos estadísticos que los estudiantes de educación secundaria del nordeste de España adquieren los conceptos e información relativa a la concienciación ecológica. 
Tabla 6: Resultados para el contraste de hipótesis unilateral donde $\mu 1$ es la media al inicio del curso y $\mu 2$ es la media al final de curso. En esta tabla se indica el curso, tipo de preguntas (total, previas o específicas de curso), el p-valor de los test-t y si este es mayor o menor al p-valor preestablecido (estadísticamente significativo cuando es $<0.05$ ).

\begin{tabular}{|c|c|c|c|}
\hline \multicolumn{4}{|c|}{ Contraste de hipótesis } \\
\hline & $\begin{array}{l}\mathrm{H}_{0}: \mu_{1} \geq \mu_{2} \\
\mathrm{H}_{\mathrm{A}}: \mu_{1}<\mu_{2}\end{array}$ & & \\
\hline Curso de educación secundaria & Tipo de preguntas & p-valor & Mayor $/$ menor $\alpha=0$ '05 \\
\hline $1^{\circ}$ & Total & 0.003203 & $<$ \\
\hline $2^{\circ}$ & Total & $1.126 \mathrm{e}-05$ & $<$ \\
\hline $2^{\circ}$ & Específicas & $2.2 \mathrm{e}-16$ & $<$ \\
\hline $2^{\circ}$ & Previas & 0.4877 & $\geq$ \\
\hline $3^{\circ}$ & Total & $9.933 \mathrm{e}-09$ & $<$ \\
\hline $3^{\circ}$ & Específicas & $4.248 \mathrm{e}-09$ & $<$ \\
\hline $3^{\circ}$ & Previas & $1.247 \mathrm{e}-05$ & $<$ \\
\hline $4^{\circ}$ & Total & 0.02522 & $<$ \\
\hline $4^{\circ}$ & Específicas & 0.6399 & $\geq$ \\
\hline $4^{\circ}$ & Previas & 0.0164 & $<$ \\
\hline
\end{tabular}

\section{Conclusión}

La evaluación de la educación secundaria es esencial para facilitar la evolución de la sociedad y de las generaciones futuras. En este estudio se ha demostrado que la educación relativa a la concienciación ecológica que reciben nuestros alumnos concluye en un aprendizaje significativo. Pese a este aprendizaje de los conceptos teóricos, no debemos descuidar la formación no formal que reciben los alumnos para así tener ciudadanos concienciados con el medio ambiente

\section{Agradecimientos}

Los autores quieren agradecerle al Dr. Sanfeliu todo el esfuerzo realizado para la consecución de este trabajo. Ha sido un pilar fundamental para el conocimiento y formación de los autores. 


\section{REFERENCIAS}

AA. VV. (1999). Libro blanco de la educación ambiental en España. Madrid, España: Ministerio de Medio Ambiente.

Alonso, B. (2010). Historia de la educacion ambiental: La Educación Ambiental en el Siglo XX.

Benayas, J., Gutiérrez, J. y Hernández, N. (2003). La investigación en educación ambiental en España. España: Ministerio de Medio Ambiente, Organismo Autónomo Parques Nacionales.

Bogner, F. X. y Wilhelm, M. G. (1996). Environmental perspectives of pupils: the development of an attitude and behaviour scale. Environmentalist, 16(2), 95-110.

Boletín Oficial del Estado. (2006a). Ley Organica 2/2006, de 3 de mayo, de Educación. España.

Boletín Oficial del Estado. (2006b). Real Decreto 1631/2006, de 29 de diciembre, por el que se establecen las enseñanzas mínimas correspondientes a la Educación Secundaria Obligatoria. España.

Boyes, E. y Stanisstreet, M. (2012). Environmental Education for Behaviour Change: Which actions should be targeted? International Journal of Science Education, 34(10), 1591-1614.

Bryan, R. R., Glynn, S. M. y Kittleson, J. M. (2011). Motivation, achievement, and advanced placement intent of high school students learning science. Science Education, 95(6), 1049-1065.

Carson, R. (1962). Silent Spring. Houghton Mifflin.

Castells, M. y Morey, M. (2004). La percepcion de los problemas ambientales y la vision del futuro en los jovenes españoles. Paper presented at the VII CONAMA, Madrid. http://www.conama.org/documentos/1763.pdf

Commoner, B. (1971). The Closing Circle. Nature, Man and Technology. Nueva York, Estados Unidos: Knopf.

Elosua, P. (2009). ¿Existe vida más allá del SPSS? Descubre R. Psicothema, 21(4), 652-655.

Garcia, J. y Nando, J. (2000). Estrategias didácticas en educación ambiental: España: Aljibe.

Gavidia, V. (2000). La construcción del concepto transversalidad. Valores y temas transversales en el curriculum (pp. 9-24).

Generalitat Valenciana. (2007). Decreto 112/2007, de 20 de julio, del Gobierno Valenciano, por el que se establece el currículo de la Educación Secundaria Obligatoria en la Comunitat Valenciana. DOGV.

Hampden-Thompson, G. y Bennett, J. (2013). Science teaching and learning activities and students' engagement in science. International Journal of Science Education, 35(8), 1325-1343.

IBM Corp. Released. (2012). SPSS. IBM Corp. SPSS Statistics for Windows. Version 21.0. Armonk, Estados Unidos: IBM Corp.

Jaén, M. y Barbudo, P. (2010). Evolución de las percepciones medioambientales de los alumnos de educación secundaria en un curso académico. Revista Eureka sobre Enseñanza y Divulgación de las Ciencias, 7.

Jiménez, M. y Lafuente, R. (2010). Defining and measuring environmental consciousness. Revista internacional de sociologia, 68(3), 731-755. doi: 10.3989/ris.2008.11.03

Meadows, D., Meadows, D., Randers, J. y Behrens, W. (1972). The limits to growth. Nueva York, Estados Unidos.

Moreno, E. (2005). La formación inicial en educación ambiental de los profesores de secundaria en periodo formativo. Valencia, España: Universidad de Valencia.

Nando, J. (1995). Detección de creencias y actitudes de los maestros de primaria y profesores de EGB en la C. Valenciana referente a la educación ambiental como elemento crítico para su implantación en el currículum. Valencia, España: Universidad de Valencia.

OECD. (2012a). Do today's 15-year-olds feel environmentally responsible? PISA, OECD Publishing. 
OECD. (2012b). Green at Fifteen? How 15-year-olds Perform in Environmental Science and Geoscience. PISA, OECD Publishing.

Peña, D. (1997). Introducción a la estadística para las ciencias sociales. Serbal.

Pérez, M. A., Pérez, M. y Quijano, R. (2009). Valoración del cambio de actitudes hacia el medio ambiente producido por el programa didáctico" EICEA" en los alumnos de Educación Secundaria Obligatoria (14-16 años). REEC: Revista electrónica de enseñanza de las ciencias, 8(3), 14.

R Core Team. (2014). R: A language and environment for statistical computing. R Foundation for Statistical Computing, Viena, Austria. Recueprado de http://www.R-project.org/

Reategui, R. (1999). Análisis crítico del modelo de enseñanza transversal en educación ambiental en la Comunidad Valenciana y propuesta para un nuevo enfoque. Valencia, España: Universidad de Valencia.

Sánchez, M. (2001). Segmentación de la población española según su grado de concienciación ecológica mediante modelos de variables latentes. Investigaciones europeas de dirección y economía de la empresa, 7(3), 173-196.

Schumacher, E. F. (1973). Small is Beautiful: Economics as If People Mattered: 25 Years Later. Hartley \& Marks Publishers.

Sierra, R. (1998). Técnicas de investigación social: teoría y ejercicios. 8222 CIC-UCAB/0191 20080723 MTiffany.

Stevens, J. (2012). Applied multivariate statistics for the social sciences. Routledge.

Tytler, R. (2012). Socio-scientific issues, sustainability and science education. Research in Science Education, 42(1), 155-163.

Vázquez-Bernal, B., Mellado, V., Jiménez-Pérez, R. y Leñero, M. (2012). The process of change in a science teacher's professional development: A case study based on the types of problems in the classroom. Science Education, 96(2), 337-363. 